\title{
Technè
}

La science au service de l'histoire de l'art et de la préservation des biens culturels

47 | 2019

Bernard Palissy : nouveaux regards sur la céramique française auX $X \mathrm{VI}^{\mathrm{e}}$ et $\mathrm{XVII}{ }^{\mathrm{e}}$ siècles

\section{De la couleur du bestiaire « esmaillé » de Palissy}

Colour in Palissy's glazed bestiary

\section{Anne Bouquillon, Gaia Ligovich et Gauthier Roisine}

\section{OpenEdition}

\section{Journals}

Édition électronique

URL : http://journals.openedition.org/techne/1494

DOI : $10.4000 /$ techne. 1494

ISSN : 2534-5168

Éditeur

C2RMF

\section{Édition imprimée}

Date de publication : 1 juin 2019

Pagination : $51-60$

ISBN : 978-2-11-152830-7

ISSN : 1254-7867

\section{Référence électronique}

Anne Bouquillon, Gaia Ligovich et Gauthier Roisine, « De la couleur du bestiaire « esmaillé » de Palissy », Technè [En ligne], 47 | 2019, mis en ligne le 01 juin 2020, consulté le 24 juillet 2020. URL : http://journals.openedition.org/techne/1494; DOI : https://doi.org/10.4000/techne.1494

\section{(ब) $\Theta \Theta$}

La revue Technè. La science au service de l'histoire de l'art et de la préservation des biens culturels est mise à disposition selon les termes de la Licence Creative Commons Attribution - Pas d'Utilisation Commerciale - Pas de Modification 4.0 International. 
Anne Bouquillon

Gaia Ligovich

Gauthier Roisine

\section{De la couleur du bestiaire « esmaillé » de Palissy}

Colour in Palissy's glazed bestiary

Résumé. La quête d'une vérité toujours plus exigeante dans la représentation de la nature caractérise l'art de Bernard Palissy. Ainsi la forme des animaux, le plus souvent aquatiques, qu'il intègre aussi bien dans ses plats de rustiques figulines que dans les éléments de grottes, est obtenue par moulage sur nature. Leurs couleurs quant à elles sont rendues par des glaçures très riches en plomb dont les nuances subtiles résultent de mélanges longuement testés. Nous en présentons ici les principales caractéristiques chimiques sur un corpus de quelques plats authentifiés et de fragments découverts dans l'atelier de Palissy, lors des fouilles des Tuileries.

Mots-clés. Palissy, glaçures plombifères, PIXE, PIGE, animaux, rustiques figulines, céramiques Renaissance.

\begin{abstract}
An ever-more demanding search for truth in the representation of nature was what characterised Bernard Palissy's art. Thus the animal forms - most frequently aquatic animals - that he integrated into both his rusticware and the ornamentation of his grottoes, were obtained by making life casts. Their colours were rendered by his use of very high lead glazes, the subtle nuances of which resulted from his long tried-and-tested mixes. Here we present the main chemical properties of the glazes on selected authenticated dishes and fragments found in Palissy's workshop during the excavations for the Grand Louvre project.

Keywords. Palissy, lead glazes, PIXE, PIGE, animals, rustiques figulines, Renaissance ceramics.
\end{abstract}

Le respect admiratif de la Création Divine (l'Homme de foi) ; la quête permanente de vérité fondée sur l'empirisme et l'observation (le scientifique) ; le perfectionnisme formel et matériel de l'« ouvrier de terre » (l'artisan) tel qu'il se définissait lui-même ; la puissance inventive (l'artiste) : ces vecteurs primordiaux de l'idiosyncrasie palisséenne éclairent le sens des énigmatiques rustiques figulines de Bernard Palissy et légitiment les techniques mises en œuvre pour leur réalisation. Il s'agit pour le céramiste de créer, en terre cuite glaçurée, des représentations animales, végétales ou minérales, en relief, les plus fidèles possibles, intégrées dans la composition de plats, d'aiguières et autres pièces de vaisselle et de parois de grottes artificielles.

En ce qui concerne la mise en forme, Palissy va privilégier le moulage sur nature, technique ancienne et déjà bien maîtrisée au Xvi ${ }^{\mathrm{e}}$ siècle, en particulier dans l'orfèvrerie allemande ${ }^{1}$. Ce numéro de Technè développe d'ailleurs quelques aspects de ce moyen d'expression ${ }^{2}$. Cependant, si les formes animales qui animent les rustiques figulines s'imposent par la vérité de leurs formes et la perfection de leur dessin, qualités obtenues par des procédés répétitifs et valorisées par le choix de leur positionnement dans les compositions, ce n'est pas tant là que se manifeste le génie du maître que dans la re-création, à partir d'oxydes métalliques traditionnels, des nuances infinies des couleurs du vivant.

Rappelons d'abord que les rustiques figulines, du moins pour les rares pièces entières qui nous sont parvenues, sont des représentations de milieux aquatiques, grouillant d'animaux marins et d'eau douce et d'environnements humides terrestres (poissons, crustacés, batraciens, reptiles, écrevisses). Ces domaines de l'eau sont des lieux de brillance, d'étincellements, de transparences colorées, bien connus du maître saintongeais. Aucun thème d'inspiration ne pouvait mieux convenir à la mise en valeur de la technique de la céramique glaçurée, passion dévorante de Palissy. D’autre part, pour lui, l'eau a toujours eu une importance essentielle dans sa réflexion scientifique, dans sa philosophie comme dans son art. Rien d'étonnant à ce que Palissy ait favorisé les mélanges glaçurants qui rendent les surfaces plus brillantes. Pour ce faire, il a utilisé de fortes teneurs en plomb (en général plus de $50 \% \mathrm{PbO}$ ) dans les formulations. Cet élément a l'avantage non seulement d'abaisser fortement les températures de fusion de la silice, mais surtout d'augmenter l'éclat des couleurs. Le céramiste a réussi de surcroît à créer des

Anne Bouquillon, ingénieur de recherche, C2RMF/IRCP (anne.bouquillon@culture.gouv.fr).

Gaia Ligovich, chimiste (gaia.ligovich@kikirpa.be). Gauthier Roisine, doctorant, C2RMF/IRCP (gauthier.roisine@laposte.net). 
surfaces plus mates et plus satinées en tirant parti de la microrugosité des reliefs, par opposition aux surfaces lisses plus brillantes, et en modifiant subtilement certaines compositions globales de glaçure. On soupçonne aussi qu'il a procédé à un traitement post-cuisson des glaçures en les corrodant volontairement par des acides, notamment pour imiter des carnations ${ }^{3}$. Les remarques qui précèdent valent pour toute la gamme colorée des rustiques figulines.

Cette attention extrême à la couleur et aux qualités des surfaces va permettre à l'artiste d'approcher au plus près l'illusion du vivant. Palissy le dit lui-même, il veut « esmaillez [ses moulages de reptiles ou de batraciens] si pres de la nature, que les autres lizers naturels et serpents, les viendront souvent admirer ${ }^{4} »$. On a retrouvé dans les fouilles du Grand Louvre nombre de petites albarelles couvertes de ses essais de glaçures, témoins des expérimentations incessantes de Palissy tout au long de sa vie de céramiste pour créer sa palette. On peut être certain que pour les rustiques figulines, chaque couleur ou presque fera l'objet d'essais préalables. Il nous dit bien les matières premières, « les drogues » comme il les appelle, qu'il utilise pour composer ses mélanges : «Les esmaux dequoy je fais ma besogne sont faits d'estaing, de plomb, de fer, d'acier, d'antimoine, de saphre de cuivre, d'arene, de salicort, de cendre gravelée, de litarge, de pierre de perigord. Voila les propres matieres desquelles je fais mes esmaux. ${ }^{5}$ " Mais il tait les dosages, les préparations préalables (calcinations, broyages...), les températures et conduites de cuisson, car « il ne faut pas divulguer légèrement des secrets que l'on acquiert à grands frais et labeurs ». « La science se manifeste à ceux qui [la] cherchent ${ }^{6}$. » Et ceux qui cherchent aujourd'hui le secret des glaçures de Palissy rencontrent bien des difficultés, même si nous avons des moyens techniques performants pour caractériser les compositions des pâtes et glaçures céramiques. Ce sont d'abord des techniques entièrement non destructives : l'une, la méthode PIXE mise en œuvre avec l'accélérateur de particules AGLAE, permet de distinguer les éléments chimiques d'une matière complexe, même ceux présents à l'état de traces ; l'autre, la diffractométrie des rayons X, aide à identifier les composants cristallisés. Quand des microprélèvements sont possibles, le microscope électronique à balayage équipé d'un détecteur de rayons X permet d'obtenir en plus les microstructures des glaçures, des pâtes et de leur interface. Les conditions analytiques précises ont été développées dans plusieurs articles et nous n'y reviendrons pas ${ }^{7}$.

Reprenons donc une à une, à la lumière des résultats obtenus par les techniques mentionnées ci-dessus, les grandes catégories des animaux figurant sur des créations considérées comme authentiquement de Palissy : le plat MR 2295 (fig. 1), l'aiguière MR 2337 (fig. 2) du musée du Louvre, le plat A 2888 du musée des Beaux-Arts de Lyon (fig. 3) et quelques tessons isolés trouvés lors des fouilles des Tuileries et conservés maintenant au musée national de la Renaissance d'Écouen (fig. 4).

Tableau 1. Compositions chimiques des glaçures transparentes

- Données PIXE et MEB exprimées en \% d'oxydes

\begin{tabular}{|l|l|l|l|l|l|l|l|l|l|l|l|l|l|l|}
\hline & $\mathrm{Na}_{2} \mathrm{O}$ & $\mathrm{MgO}$ & $\mathrm{Al}_{2} \mathrm{O}_{3}$ & $\mathrm{SiO}_{2}$ & $\mathrm{SO}_{3}$ & $\mathrm{Cl}$ & $\mathrm{K}_{2} \mathrm{O}$ & $\mathrm{CaO}$ & $\mathrm{KiO}_{2}$ & $\mathrm{MnO}^{-}$ & $\mathrm{Fe}_{2} \mathrm{O}_{3}$ & $\mathrm{CuO}$ & $\mathrm{SnO}_{2}$ & $\mathrm{Pb0}$ \\
\hline $\begin{array}{l}\text { 121.002.17 coquille jaune } \\
\text { R 217 coquille } \\
\text { transparente blanche }\end{array}$ & 0,22 & 0,23 & 3,77 & 35,86 & 0,65 & 0,28 & 0,88 & 2,78 & 0,21 & 0,02 & 1,09 & 0,06 & 0,03 & 53,83 \\
\hline $\begin{array}{l}\text { MR 2295 coquille } \\
\text { transparente blanche }\end{array}$ & 1,26 & 0,47 & 0,99 & 43,61 & 0,11 & 0,29 & 1,15 & 1,32 & 0,1 & 0,18 & 0,26 & 0,06 & 0 & 50,05 \\
\hline $\begin{array}{l}\text { MR 2337 coquille } \\
\text { transparente blanche }\end{array}$ & 1,30 & 0,37 & 0,89 & 53,70 & 0,00 & 0,27 & 0,69 & 1,75 & 0,11 & 0,17 & 0,61 & 0,08 & 0,63 & 39,35 \\
\hline MR 2337 coquille jaune & 0,85 & 0,96 & 4,31 & 40,26 & 0,15 & 0,18 & 0,89 & 6,26 & 0,42 & 0,33 & 1,96 & 0,14 & 0,19 & 42,98 \\
\hline
\end{tabular}

Tableau 2. Épaisseurs moyennes de glaçures mesurées au MEB sur des coupes stratigraphiques

\begin{tabular}{|l|l|l|}
\hline Animaux & $N^{\circ}$ d'inventaire & Épaisseurs \\
\hline Coquille jaune & \multirow{2}{*}{121.002 .17} & $80-140 \mu \mathrm{m}$ (sur 3 prélèvements) \\
\cline { 1 - 1 } Coquille blanche & & $50-70$ (sur 3 prélèvements) \\
\hline Lézard brun & \multirow{2}{*}{ AC. 243.1585} & 55 \\
\cline { 3 - 3 } Lézard vert & & 55 \\
\hline Grenouille verte & EP 347 & 70 \\
\hline
\end{tabular}




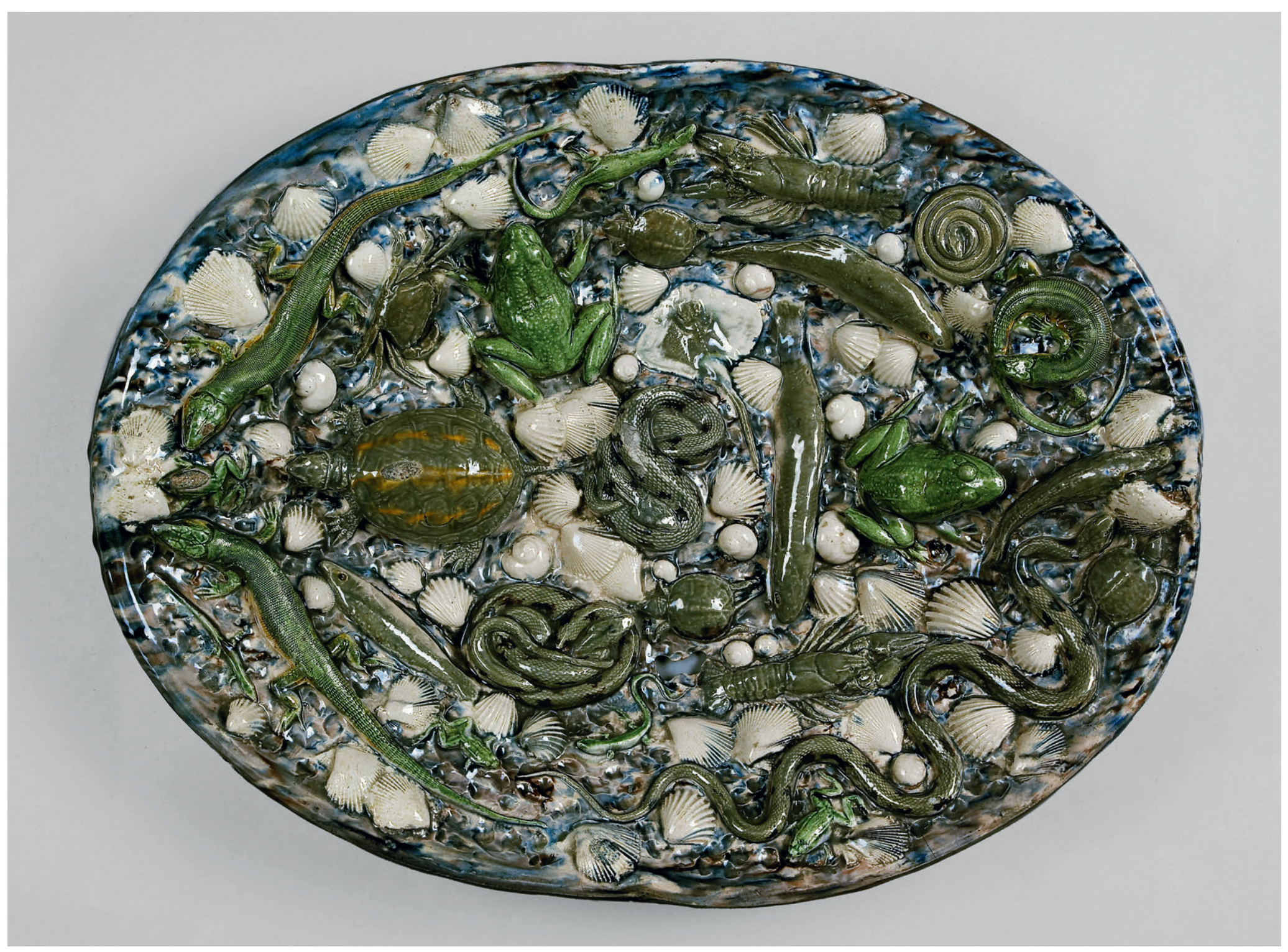

Fig. 1. Bernard Palissy, Saintes (?), vers 1555-1565, Plat à décor de rustiques figulines,

terre cuite glaçurée (L. $48 \mathrm{~cm}, 1.37 \mathrm{~cm}$, H. 9,5 cm), Paris, musée du Louvre, MR 2295. () C2RMF/D. Bagault. 


\section{Analyses des différentes couleurs}

\section{Le blanc et le jaune des coquilles}

Les coquilles sont d'origines marines variées (pétoncles, buccins, praires, murex....) - sans aucun fossile, ce qui caractérise les productions authentiques de Palissy (voir l'article de J-C. Plaziat dans ce volume) ; elles ont la couleur blanche ou ivoire qui est la leur dans la nature. Pour la mise en couleur de ces coquillages, le céramiste recouvre la pâte argileuse très fine, blanche, kaolinitique du support, d'une glaçure transparente, absolument incolore pour les blancs et légèrement enrichie en fer pour les plus jaunes. L'analyse chimique de la couverte (tableau 1) montre qu'il n'y a ici pratiquement pas fondant principal ; dans les glaçures des coquilles plus jaunes, l'augmentation très subtile du fer, souvent associée à de plus fortes teneurs en aluminium et titane, suggère que le céramiste a intégré des petites quantités d'argiles, suffisamment faibles et dans une granulométrie assez fine pour que la transparence de la glaçure n'en soit pas affectée. Il n’y a pas de glaçure blanche opaque. Aucune trace d'opacification par la cassitérite pour intensifier la couleur blanche n'a été identifiée. La présence dans certaines coquilles jaunes de quantités plus élevées de calcium, apportées soit par l'argile, soit par ajout volontaire de calcite, pourrait avoir eu un léger effet matifiant sur le brillant de la glaçure sans modifier la transparence, mais c'est, pour le moment, très difficile à confirmer. Par ailleurs, la coloration jaune pâle n'apparaîtrait pas si la couche vitrifiée était insuffisamment épaisse. Elle mesure ici $140 \mu \mathrm{m}$ pour les coquilles jaunes, alors qu'elle varie entre 50 et $80 \mu \mathrm{m}$ pour les transparentes (tableau 2).

\section{Le vert des batraciens}

La plupart des grenouilles sont vertes. La peau gluante est parfaitement rendue par une glaçure colorée avec des oxydes de cuivre, mais on distinguera deux catégories : les compositions avec ou sans étain, c'est-à-dire les glaçures transparentes ou opacifiées. Les teneurs en oxydes métalliques colorants ne changent pas : pour le cuivre de 2,4 à $3 \%$ $\mathrm{CuO}$, le fer reste voisin de $0,5 \% \mathrm{Fe}_{2} \mathrm{O}_{3}$. Palissy utilise un cuivre

non allié pour réaliser son pigment, ce qui le différencie de la plupart des céramistes postérieurs, chez lesquels le cuivre est associé au zinc (laiton), ou à l'étain (bronze), suggérant l'utilisation de résidus d'alliages à base cuivre (tableau 3).

En revanche, Palissy joue sur les proportions d'étain pour obtenir des qualités de verts différentes. Quand il n'y en a pas, comme dans les grenouilles étudiées par Isabelle Perrin ${ }^{8}$ et dans un ensemble de grenouilles découvertes lors des fouilles et vraisemblablement attribuées à Palissy ${ }^{9}$, la couleur est translucide (fig. 4). Mais dans les plats et l'aiguière, le vert est opacifié à l'étain dans des proportions qui varient de 1 (grenouille du plat de Lyon A 2888) à 3 et $5 \% \mathrm{SnO}_{2}$ pour les grenouilles de l'aiguière et du plat du Louvre. On sait que ces concentrations sont suffisantes pour assurer une opacité, du moins une opalescence à un verre. Il y a là une recherche évidente d'effets particuliers. Ces variations sont parfois difficiles à repérer à l'œil nu. Un des tessons découverts lors des fouilles du Louvre (NP3), mais correspondant probablement à une vaisselle ${ }^{11}$ montre à cet égard toute la virtuosité de l'artiste : on passe d'un vert quasi transparent $\left(2 \% \mathrm{SnO}_{2}\right)$ à un vert totalement opaque plus sombre à $24 \% \mathrm{SnO}_{2}$ sans qu'il n'y ait de modification dans la qualité de la glaçure. Nous avions aussi pensé que Palissy aurait pu utiliser un pigment jaune pour aviver certains verts, comme le faisaient à l'époque les artistes italiens (par exemple les Della Robbia ou les peintres de majoliques comme Xanto Avelli), en ajoutant des antimoniates de plomb, jaunes, au mélange. Ici, il aurait pu s'agir d'un jaune de plomb et d'étain : à la différence de ce qui est mis en œuvre dans les palettes des peintres, ce pigment est assez rare dans le domaine de la céramique, mais il a été détecté par microspectrométrie Raman dans le brun d'un des médaillons palisséens découverts lors des fouilles ${ }^{12}$. Cependant, l'observation par microscopie électronique à balayage d'une fine écaille de glaçure verte n'a pas permis de confirmer cette hypothèse pour le moment.

\section{Le vert des lézards}

Tout comme pour les grenouilles, les lézards présentent une couleur verte uniforme : l'oxyde de cuivre est le principal élément colorant (3,2\% CuO en moyenne), il n'est pas associé au zinc, au nickel ou à l'arsenic. Il semble donc que l'origine de ce pigment soit là aussi un cuivre assez pur. En tout cas, la

Tableau 3. Compositions chimiques de différentes glaçures vertes - Données PIXE exprimées en \% d'oxydes

\begin{tabular}{|l|l|l|l|l|l|l|l|l|l|l|l|l|l|l|l|l|l|l|}
\hline & $\mathrm{Na}_{2} \mathrm{O}$ & $\mathrm{MgO}$ & $\mathrm{Al}_{2} \mathrm{O}_{3}$ & $\mathrm{SiO}_{2}$ & $\mathrm{SO}_{3}$ & $\mathrm{Cl}$ & $\mathrm{K}_{2} \mathrm{O}$ & $\mathrm{CaO}$ & $\mathrm{TiO}_{2}$ & $\mathrm{MnO}$ & $\mathrm{Fe}_{2} \mathrm{O}_{3}$ & $\mathrm{CoO}$ & $\mathrm{NiO}$ & $\mathrm{CuO}$ & $\mathrm{ZnO}$ & $\mathrm{As}_{2} \mathrm{O}_{3}$ & $\mathrm{SnO}_{2}$ & $\mathrm{PbO}$ \\
\hline EP 347 grenouille verte & 0,63 & 0,85 & 8,54 & 30,05 & 0,00 & 0,30 & 0,40 & 1,87 & 0,58 & 0,03 & 0,53 & 0,01 & 0,02 & 2,85 & 0,02 & 0,00 & 0,00 & 53,24 \\
\hline MR 2295 grenouille verte & 0,68 & 1,02 & 10,78 & 32,04 & 0,00 & 0,24 & 0,41 & 0,92 & 0,51 & 0,02 & 0,56 & 0,01 & 0,01 & 2,55 & 0,03 & 0,05 & 3,60 & 46,51 \\
\hline MR 2337 grenouille verte & 0,56 & 0,69 & 7,41 & 31,38 & 0,00 & 0,10 & 0,30 & 0,80 & 0,56 & 0,00 & 0,44 & 0,01 & 0,01 & 2,81 & 0,01 & 0,00 & 4,42 & 50,40 \\
\hline MR 2295 lézard vert & 0,63 & 1,02 & 9,43 & 27,13 & 0,00 & 0,22 & 0,32 & 0,52 & 0,49 & 0,01 & 0,70 & 0,01 & 0,02 & 3,27 & 0,03 & 0,01 & 4,13 & 52,08 \\
\hline AC. 243.1515 corps lézard & 1,24 & 0,86 & 5,43 & 21,42 & 0,17 & 0,68 & 1,27 & 1,87 & 0,57 & 0,08 & 0,62 & 0,01 & 0,02 & 2,98 & 0,02 & 0,00 & 14,02 & 48,64 \\
\hline
\end{tabular}


présence d'une rugosité importante créée par les écailles contrastant avec la peau relativement plus lisse des grenouilles donne une impression colorée autre, due à la diffraction différente de la lumière sur les surfaces : la peau des lézards paraît souvent plus sombre et plus mate. Il faut aussi noter que, pour ne pas effacer le dessin des écailles, Palissy applique une couche vitreuse particulièrement fine, mesurée sur un des tessons à $50 \mu \mathrm{m}$ (tableau 2). La présence d'étain est systématique, à la différence des grenouilles, aux alentours de $5 \%$ $\mathrm{SnO}_{2}$ pour les deux lézards du plat et beaucoup plus élevée pour le fragment de patte du tesson AC. 243.1515 (13\% $\mathrm{SnO}_{2}$ ). Cependant, la prudence s'impose ici : en effet, le fragment a été enfoui et il est fortement altéré. Des recherches sur d'autres fragments palisséens ont montré, par l'analyse des microstructures, que lors de l'altération d'un verre opaque, les cristaux de cassitérite se concentrent dans la couche altérée, entraînant ainsi une augmentation artificielle des teneurs en étain en surface ${ }^{13}$. Rappelons ici que nous avons travaillé avec l'accélérateur de particules pour limiter les prélèvements et que cette technique permet d'explorer les premières dizaines de micromètres de la surface, là où les perturbations liées aux altérations d'enfouissement sont les plus fortes. Quoi qu'il en soit, les verts des lézards analysés jusqu'à présent sont tous opacifiés, ce qui n'est pas le cas pour les grenouilles.

Un autre aspect est également à souligner, commun à toutes les glaçures vertes. L'oxyde de cuivre peut agir comme un fondant efficace ; associé au plomb, il abaisse notablement la température de fusion. Les parties colorées en vert sont donc susceptibles, lors de la cuisson, de fondre plus vite et de couler sur les autres. Palissy le dit bien : « Ayant fait un certain nombre de bassins rustiques \& les ayant fait cuire, mes esmaux se trouvoyent les uns beaux \& bien fonduz, autres mal fonduz, autres estoyent brulez, à cause qu'ils estoyent composez de diverses matieres qui estoyent fusibles à divers degrez, le vert des lezards estoit bruslé premier que la couleur des serpens fut fondue, aussi la couleur des serpens, escrevices, tortues \& cancres, estoit fondue au paravant que le blanc eut reçeu aucune beauté ${ }^{14}$. »

Tous les verts sont systématiquement plus riches en aluminium que les autres couleurs. Des expérimentations d'ajouts croissants d'aluminium dans un mélange silice, plomb, cuivre montrent que cet élément n'a aucune influence sur la couleur ${ }^{15}$. On pense que Palissy a pu volontairement ajouter de l'aluminium, réfractaire, apporté par des argiles (la kaolinite contient $40 \%$ de $\mathrm{Al}_{2} \mathrm{O}_{3}$ dans sa composition) pour contrecarrer les effets fondants du cuivre et pour contrôler la viscosité du mélange. Il fallait que ces glaçures vertes fondent, nappent la surface sans se mélanger aux autres dans les mêmes gammes de température, dans les mêmes atmosphères et, si l'on en croit ce qu'il écrit, en une seule cuisson après le dégourdi : un vrai «tour de force " comme l'a défini A. Heuer en 2008 lors d'une conférence au musée de Cleveland sur « Palissy ingénieur ».

Il est étonnant de constater jusqu'à présent l'absence des mélanges d'oxydes utilisés habituellement par les céramistes italiens de la même époque : ici, apparemment, le maître n'a pas joué sur les rapports fer/cuivre ou manganèse/cuivre, voire cobalt/cuivre qui pourraient donner des verts plus sombres, plus émeraude. On notera aussi dans les albarelles

Tableau 4. Compositions chimiques de différentes glaçures grises - Données PIXE exprimées en \% d'oxydes

\begin{tabular}{|l|l|l|l|l|l|l|l|l|l|l|l|l|l|l|l|l|l|l|l|}
\hline & $\mathrm{Na}_{2} \mathrm{O}$ & $\mathrm{MgO}$ & $\mathrm{Al}_{2} \mathrm{O}_{3}$ & $\mathrm{SiO}_{2}$ & $\mathrm{SO}_{3}$ & $\mathrm{Cl}$ & $\mathrm{K}_{2} \mathrm{O}$ & $\mathrm{CaO}$ & $\mathrm{TiO}_{2}$ & $\mathrm{MnO}$ & $\mathrm{Fe}_{2} \mathrm{O}_{3}$ & $\mathrm{CoO}$ & $\mathrm{NiO}$ & $\mathrm{CuO}$ & $\mathrm{ZnO}$ & $\mathrm{As}_{2} \mathrm{O}_{3}$ & $\mathrm{SnO}_{2}$ & $\mathrm{PbO}$ & $\mathrm{Sb}_{2} \mathrm{O}_{5}$ \\
\hline 121.133.64 serpent & 0,92 & 0,55 & 4,84 & 25,63 & 5,52 & 0,44 & 1,77 & 7,88 & 0,48 & 2,00 & 2,52 & 0,15 & 0,06 & 0,22 & 0,04 & 0,01 & 4,36 & 41,49 & 0,12 \\
\hline EP 343 serpent & 0,97 & 0,59 & 4,03 & 26,65 & 0,07 & 0,44 & 0,49 & 2,74 & 0,46 & 0,79 & 2,34 & 0,12 & 0,06 & 0,33 & 0,03 & 0,23 & 6,60 & 52,93 & 0,12 \\
\hline AC. 243.1860 serpent & 1,59 & 0,61 & 4,52 & 45,53 & 0,68 & 0,49 & 5,09 & 4,39 & 0,28 & 3,07 & 4,02 & 0,25 & 0,07 & 0,68 & 0,12 & 0,20 & 9,38 & 18,82 & 0,09 \\
\hline
\end{tabular}

Tableau 5. Compositions chimiques de glaçures brunes - Données PIXE exprimées en \% d'oxydes

\begin{tabular}{|c|c|c|c|c|c|c|c|c|c|c|c|c|c|c|c|c|c|c|c|}
\hline & $\mathrm{Na}_{2} \mathrm{O}$ & $\mathrm{MgO}$ & $\mathrm{Al}_{2} \mathrm{O}_{3}$ & $\mathrm{SiO}_{2}$ & $\mathrm{SO}_{3}$ & $\mathrm{Cl}$ & $\mathrm{K}_{2} \mathrm{O}$ & $\mathrm{CaO}$ & $\mathrm{TiO}_{2}$ & $\mathrm{MnO}$ & $\mathrm{Fe}_{2} \mathrm{O}_{3}$ & CoO & $\mathrm{NiO}$ & CuO & $2 \mathrm{no}$ & $\mathrm{As}_{2} \mathrm{O}_{3}$ & $\mathrm{SnO}_{2}$ & $\mathrm{PbO}$ & $\mathrm{P}_{2} \mathrm{O}_{5}$ \\
\hline EP 820 tortue brun & 0,72 & \begin{tabular}{|l|l|}
0,51 \\
\end{tabular} & 3,62 & 19,13 & 1,00 & 0,54 & 0,03 & 2,22 & 0,56 & 0,44 & 2,47 & 0,13 & 0,06 & 0,36 & 0,01 & 0,30 & 13,03 & 54,68 & 0,00 \\
\hline $\begin{array}{l}\text { EP } 820 \text { tortue brun } \\
\text { tache jaune }\end{array}$ & 1,05 & 0,55 & 3,82 & 17,92 & 1,09 & 0,66 & 0,09 & 1,94 & 0,62 & 1,66 & 2,41 & 0,15 & 0,07 & 0,52 & 0,03 & 0,18 & 12,79 & 54,40 & 0,00 \\
\hline \multirow[t]{2}{*}{ MR 2295 jaune tortue } & 0,31 & 0,24 & 4,11 & 20,55 & 0,00 & 0,00 & 0,00 & 0,00 & 0,48 & 0,14 & 2,75 & 0,05 & 0,04 & 0,21 & 0,01 & 0,18 & 10,75 & 60,18 & 0,00 \\
\hline & $\mathrm{Na}_{2} \mathrm{O}$ & $\mathrm{MgO}$ & $\mathrm{Al}_{2} \mathrm{O}_{3}$ & $\mathrm{SiO}_{2}$ & $\mathrm{SO}_{3}$ & $\mathrm{Cl}$ & $\mathrm{K}_{2} \mathrm{O}$ & $\mathrm{CaO}$ & $\mathrm{TiO}_{2}$ & $\mathrm{MnO}$ & $\mathrm{Fe}_{2} \mathrm{O}_{3}$ & CoO & NiO & CuO & $\mathrm{ZnO}$ & $\mathrm{As}_{2} \mathrm{O}_{3}$ & $\mathrm{SnO}_{2}$ & $\mathrm{PbO}$ & $\mathrm{BaO}$ \\
\hline R 217 buvant brun & 0,77 & 0,35 & 4,47 & 27,52 & 0,03 & 0,38 & 0,73 & 0,60 & 0,24 & 0,06 & 3,54 & 0,01 & 0,02 & 0,07 & 0,01 & 0,00 & 5,11 & 56,09 & 0,00 \\
\hline $\begin{array}{l}\text { MR } 2337 \text { écrevisse } \\
\text { plus clair }\end{array}$ & 1,15 & 0,66 & 4,23 & 40,26 & 0,00 & 0,17 & 0,70 & 2,47 & 0,32 & 0,84 & 1,97 & 0,18 & 0,07 & 0,61 & 0,02 & 0,03 & 7,85 & 38,39 & 0,07 \\
\hline MR 2337 écrevisse & 2,09 & 0,69 & 3,43 & 33,09 & 0,00 & 0,70 & 0,79 & 2,23 & 0,29 & 0,69 & 1,68 & 0,17 & 0,07 & 0,87 & 0,03 & 0,12 & 9,04 & 43,96 & 0,08 \\
\hline $\begin{array}{l}\text { MR } 2337 \text { pince brune } \\
\text { de l'écrevisse }\end{array}$ & 1,15 & 0,58 & 3,86 & 27,18 & 0,00 & 0,34 & 0,56 & 1,80 & 0,49 & 3,67 & 1,69 & 0,19 & 0,07 & 0,74 & 0,03 & 0,10 & 13,74 & 43,55 & 0,23 \\
\hline
\end{tabular}


Fig. 2. Bernard Palissy, Saintes (?), vers 1555-1565, Aiguière à décor de rustiques figulines, terre cuite glaçurée (H. 19,5 cm, L. $19 \mathrm{~cm}$, 1. $14 \mathrm{~cm}$ ), Paris, musée du Louvre, MR 2337. ( $)$ RMN-Grand Palais (musée du Louvre) /Jean-Gilles Berizzi.

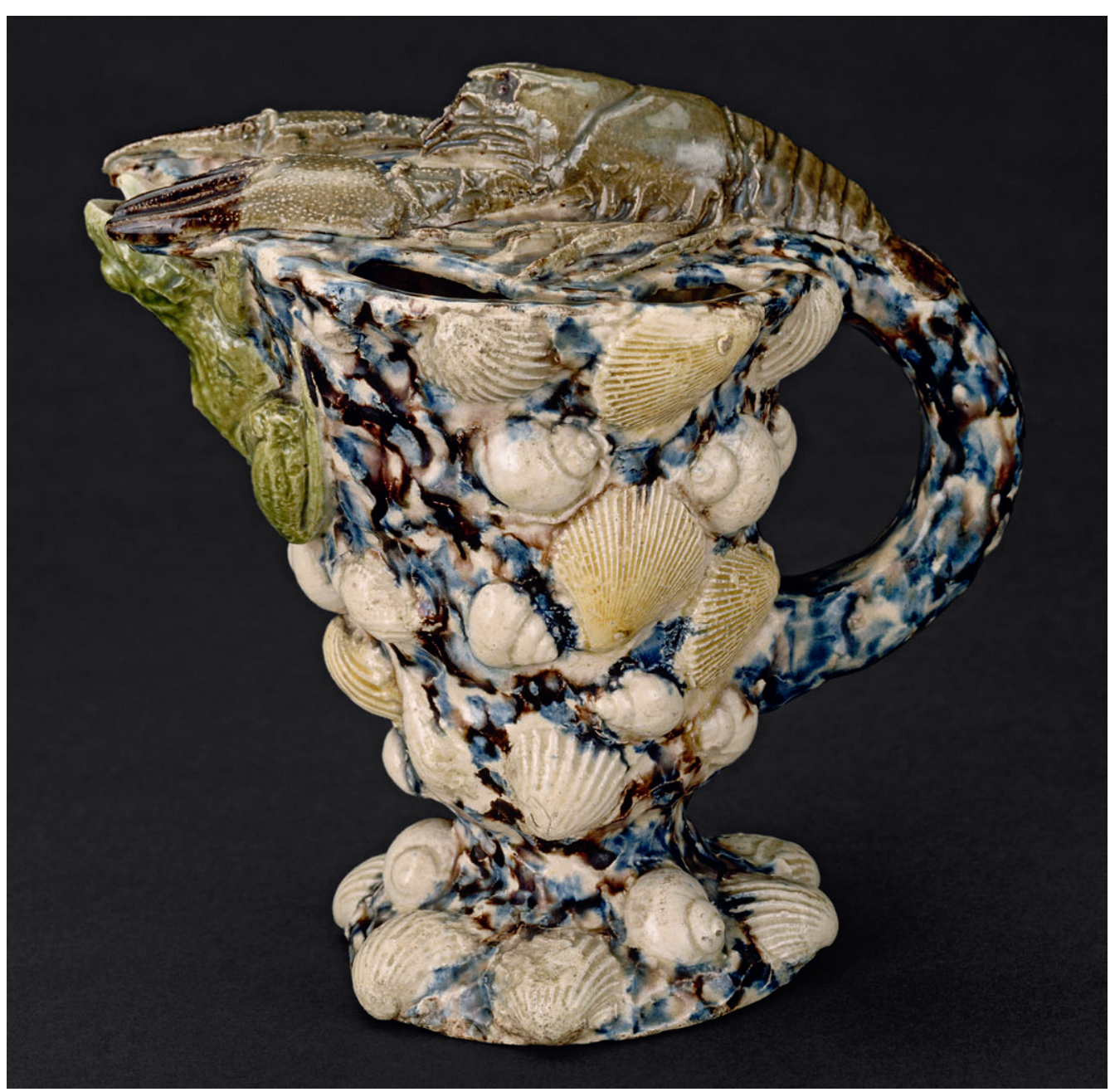

qui nous sont parvenues (fig. 5) une quasi absence d'essais de verts. Peut-être Palissy n'a-t-il pas éprouvé le besoin d'aller plus loin dans la recherche des nuances de la palette des verts.

\section{Poissons et reptiles gris}

Sur les gris des reptiles et des poissons, les glaçures sont encore différentes. Le ton est obtenu par un mélange de fer $\left(1,5\right.$ à $\left.2 \% \mathrm{Fe}_{2} \mathrm{O}_{3}\right)$, de cuivre $(0,5 \% \mathrm{CuO})$, mais surtout de cobalt $(0,2 \% \mathrm{CoO}$ en moyenne) et de manganèse $(0,5 \%$ $\mathrm{MnO}$ en moyenne). Les analyses n’ont pas fait apparaître de fortes variations dans les proportions relatives de ces différents oxydes métalliques. C'est une fois encore dans les teneurs en étain, lequel se trouve sous forme de cassitérite, que les variations sont les plus nettes (tableau 4) : il est présent jusqu'à une proportion de $10 \% \mathrm{SnO}_{2}$ pour le poisson alors qu'elle n'est que de $7 \% \mathrm{SnO}_{2}$ pour la couleuvre centrale. Il semble bien que Palissy cherche à jouer autant sur les degrés d'opacification et l'intensité de la teinte que sur des mélanges complexes d'oxydes. Pour les potiers du xvi ${ }^{\mathrm{e}}$ siècle, le gris doit être une couleur certainement compliquée à obtenir, en témoignent les nombreux essais réalisés sur des albarelles. On trouve en effet très peu de gris sur les terres cuites glaçurées robbiesques, sauf dans certains détails d'œuvres de Giovanni della Robbia par exemple.

\section{Reptiles et crustacés bruns}

La même influence de l'étain se retrouve dans les teintes brunes, qu'il s'agisse de serpents ou de crustacés (écrevisses, crabes, etc.). Prenons l'exemple de l'écrevisse de l'aiguière MR 2337 (fig. 2) : le corps est d'un brun rosé assez clair, l'extrémité des pinces est d'un marron rouge plus dense. À chaque fois, les mélanges colorés sont constitués des mêmes oxydes métalliques, mais les proportions sont très différentes : le fer est dominant dans le corps $\left(2 \% \mathrm{Fe}_{2} \mathrm{O}_{3}\right.$ pour $0,8 \%$ $\mathrm{MnO})$, le manganèse est plus élevé dans les pinces $(3,5 \%$ $\mathrm{MnO}$ pour $1,7 \% \mathrm{Fe}_{2} \mathrm{O}_{3}$ ), cobalt et cuivre restent constants 
(respectivement $0,2 \% \mathrm{CoO}$ et $0,7 \% \mathrm{CuO}$ ), tandis que les teneurs en étain changent $\left(8 \% \mathrm{SnO}_{2}\right.$ pour le corps, plus de $13 \%$ dans les pinces) (tableau 5 ).

Les nuances de brun sont obtenues en modulant les proportions de fer (de 1,5 à $\left.4 \% \mathrm{Fe}_{2} \mathrm{O}_{3}\right)$ et de manganèse (plutôt voisines de $0,5 \%$ pour du brun clair, supérieures à 2,5\% MnO pour les nuances les plus sombres), auxquelles sont ajoutées des proportions d'étain extrêmement variables de 2 à $15 \% \mathrm{SnO}_{2}$.

Si l'on considère plus particulièrement ces teintes grises ou brunes, sur un même animal, on met en évidence des variations très nettes de concentrations entre des points d'analyses rapprochés, en particulier sur la tortue bicolore (gris et jaune de la carapace) ou les serpents tachetés. Cela signifierait que Palissy a travaillé la couleur par touches. Sa formation de peintre-verrier n'est certainement pas étrangère à ce savoir-faire. De très nombreux essais de glaçures grises ou brunes ont été découverts sur les albarelles, certains totalement ratés, d'autres pulvérulents ou partiellement vitrifiés, d'autres encore parfaitement couvrants. Les recherches sont en cours sur ces essais.

Palissy utilise également d'autres techniques pour modifier les apparences colorées des glaçures transparentes sans changer le mélange vitreux. C'est la couleur de la pâte sousjacente qui joue : c'est ainsi qu'il passe d'un jaune pâle à un orangé brun en ajoutant sous la zone qui doit être la plus foncée une fine couche d'engobe d'argile rouge. Ce savoirfaire est surtout observé dans les fragments de parois de grotte.

\section{Savoir-faire et innovations}

Cette étude encore très parcellaire de la couleur des animaux dans l'œuvre de l'inventeur des rustiques figulines montre que le métier de ce génial « ouvrier de terre » et son art céramique sont exceptionnels : aucun autre artiste avant lui n'avait osé utiliser des compositions si différentes sur un même support, ni n'avait réussi à rendre les « divers émaux fusibles à un même degré de feu ${ }^{16}$ ». "Le verd des lezards estoit bruslé premier que la couleur des serpens fut fondue ${ }^{17}$. » Une étude approfondie des glaçures miel réalisée par G. Roisine dans le cadre de sa thèse, même si ce n'est pas sur des représentations d'animaux mais plutôt des fragments de vaisselle ou de décors architecturaux, montre que Palissy utilise les dosages les plus réfléchis pour obtenir la couleur la plus dense en préservant la transparence absolue sans empâter les reliefs. Il dose exactement le fer qu'il ajoute dans le mélange glaçurant, ni trop, ni trop peu et, quand les concentrations en fer dépassent $3,5 \% \mathrm{Fe}_{2} \mathrm{O}_{3}$, cela est probablement dû à l'engobe rouge systématiquement trouvé sous ces glaçures riches en fer. Ces glaçures, vraisemblablement fortuitement enrichies en fer, peu nombreuses à l'échelle de la production palisséenne, sont caractérisées par la présence d'hématite, indice laissé par Palissy bien malgré lui, et qui témoigne de sa grande maîtrise. Il réussit à maîtriser les températures du four dans un intervalle de $50{ }^{\circ} \mathrm{C}$ autour de $925^{\circ} \mathrm{C}^{18}$, juste dans la fenêtre de température dans laquelle les cristaux de première génération ont disparu et à une température inférieure à celle où cristallisent d'autres composés fer/plomb. Ces considérations sont valables pour les glaçures miel transparentes, mais on peut être certain que Palissy a travaillé toutes les autres couleurs (et elles sont nombreuses dans les œuvres ici étudiées) pour qu'elles obéissent aux mêmes impératifs de la cuisson.

Les céramistes post-palisséens ont-ils bénéficié de toute cette expérience ? Si l'on prend l'exemple de leurs rustiques figulines, pendant longtemps, même jusqu'au XIx ${ }^{\mathrm{e}}$ siècle, un seul type de glaçure recouvre ces plats. Il s'agit le plus souvent d'une glaçure plombifère transparente ; ce sont des ajouts d'oxydes métalliques colorants qui donnent la palette ${ }^{19}$. On n'y trouve pas de recherche d'effet entre l'opaque, le semiopaque et le transparent, ni de travail sur la couleur de la pâte ou de l'engobe sous-jacent. En aucun cas, l'étain n'y joue le rôle stratégique que nous avons mis en évidence dans les productions palisséennes.

Ce qui est sûr, c'est que, même si l'on trouve chez certains créateurs de la Renaissance italienne comme les Della Robbia des exemples de fruits et même d'animaux en relief dans des compositions en terre cuite glaçurée ${ }^{20}$, rien n'approche le degré de vérité atteint par Bernard Palissy dans le rendu des attitudes, des textures et des couleurs des animaux.

On célèbre avec raison le naturalisme mimétique de Palissy et on ne peut nier que chaque représentation animale prise séparément approche vraiment de la réalité du vivant comme le désirait l'artiste dans sa célébration de la création divine, mais les compositions et les regroupements de ces animaux dans ses œuvres appellent d'autres remarques. En effet, quand on regarde un bassin de rustiques figulines, ce n'est pas le qualificatif «vrai » qui vient le premier à l'esprit ; mais bien celui de « bizarre », "d'invraisemblable », et c'est bien là l'effet que produit ce grouillement d'êtres vivants (fig. 1). Cette impression se renforce à l'étude quand on se rend compte qu'il est composé d'animaux dont les biotopes sont incompatibles : le grondin et la raie ne vivent pas avec les écrevisses, ni les couleuvres avec les bulots !! Il est tentant de chercher à établir des rapprochements avec certains détails de l'Eau ${ }^{21}$ d'Arcimboldo, exact contemporain de Palissy. Chez ce dernier toutefois, ni intention fantastique ni caricature, il exalte la vie et célèbre la richesse, la variété du monde.

La composition générale semble bien obéir à une répartition organisée des formes et des couleurs (fig. 3), mais celleci est assurément toute subjective. La sensibilité de l'artiste l'emporte sur la réalité. Dans cet aspect précis de l'œuvre, on est loin de l'imitation pure ; la Nature se plie à la créativité de Palissy, créativité qui s'exprime aussi bien dans l'exubérance de la composition que dans le choix des tons rares et élaborés dans la palette infinie des couleurs de la Nature, sans intensité agressive.

En effet, en ce qui concerne la couleur, ce n'est pas, comme pour les céramistes des époques précédentes et même pendant tout le temps des céramistes post-palisséens, la couleur apprise, ordinaire, plus ou moins nuancée, que l'on restitue au besoin avec des mélanges simples d'oxydes 


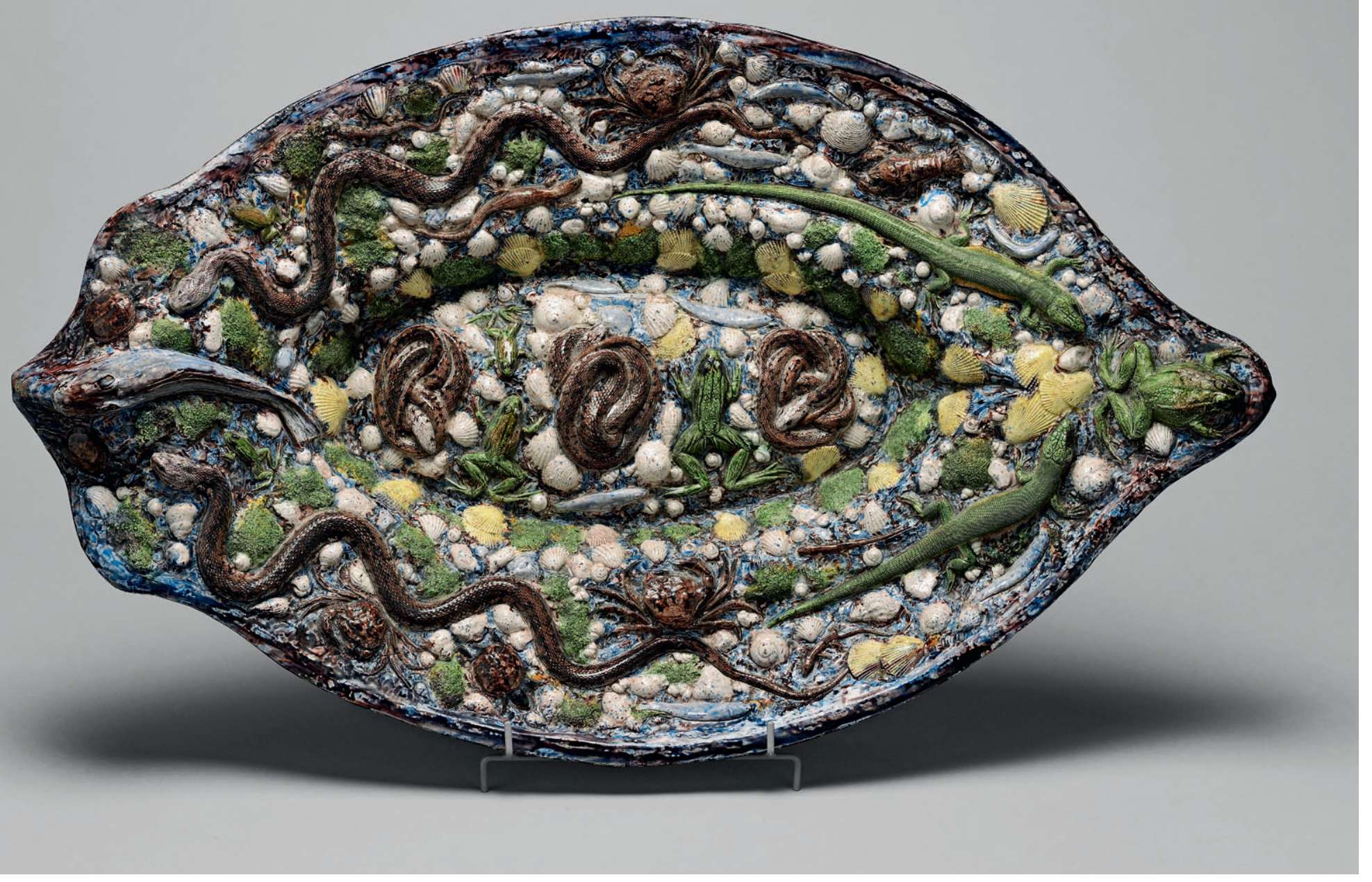

Fig. 3. Bernard Palissy, Saintes (?), vers 1555-1565, Plat à décor de rustiques figulines,

terre cuite glaçurée (L. 45 cm, 1. 74,6 cm, H. 16,9 cm), Lyon, musée des Beaux-Arts, A 2888.

(c) Musée des Beaux-Arts de Lyon. 
colorants traditionnels, c'est la couleur éprouvée par l'artiste devant chaque modèle, dans son unicité, qu'il faut saisir par tous les moyens, les compositions complexes, comme les prouesses technologiques, car la couleur des choses est fugace comme le dit Palissy dans les Discours admirables : "Il n'y a nulle chose sous le ciel en repos, \& que toutes choses se travaillent en se formant, \& en se deformant tournent bien souvent de nature à autre, \& et de couleur à autre ${ }^{22}$. » Ne peut-on voir une illustration de cette observation dans les deux plats H 475 (voir fig. 3 de l'article de P. H. Smith dans ce volume) et A 2888 du musée des Beaux-Arts de Lyon (fig. 3), semblables mais de couleurs différentes, au point que d'une pièce à l'autre les serpents semblent avoir changé de peau et tout l'environnement de nuances?

Sur les rustiques figulines, l'absolue précision atteinte par les glaçures dans la re-création des couleurs de la faune des milieux humides et aquatiques est un témoignage parmi d'autres, nombreux et très différents, de la passion que Palissy met à lire la Nature. Cette lecture n'est pas, chez lui, qu'une contemplation admirative de la création divine. Le croyant se double ici, chez cet autodidacte, d'un grand scientifique, ardent à retrouver par l'expérimentation les mécanismes de la formation de toute chose, afin de pouvoir imiter la Nature et multiplier ainsi les trésors qu'elle nous prodigue ; car vivre avec philosophie, c'est travailler à « aider la nature ${ }^{23}$ » écrit-il dans les Discours admirables.

Les domaines dans lesquels Palissy exerce son esprit d'analyse et de déduction sont multiples, depuis l'agriculture, la chimie, jusqu'à l'action des sels, en passant par l'hydrologie ; mais c'est en tant que potier, " ouvrier de terre ", qu'il se définit lui-même, lui qui va consacrer la majeure partie de son activité aux arts du feu, en particulier aux secrets des glaçures colorées, non pas tellement les couleurs pures, de compositions habituelles, mais celles de la nature dont il a besoin pour nourrir son ouvre d'artiste, entre imitation, invention et illusion. On ne s'étonnera pas alors que ses expérimentations dans ce domaine aient porté en grande partie sur les gris. Les rustiques figulines, dans leur aspect et à travers l'analyse physico-chimique des composants, en sont une illustration frappante.

Le bestiaire des rustiques figulines, même considéré sous le seul aspect de la couleur, ouvre ainsi sous l'angle de la philosophie, de la technique, de la science et de l'art, des perspectives si vastes et si nombreuses qu'on ne peut que célébrer Palissy comme un novateur, un «phare " tout à fait unique, pour ne pas dire inclassable.

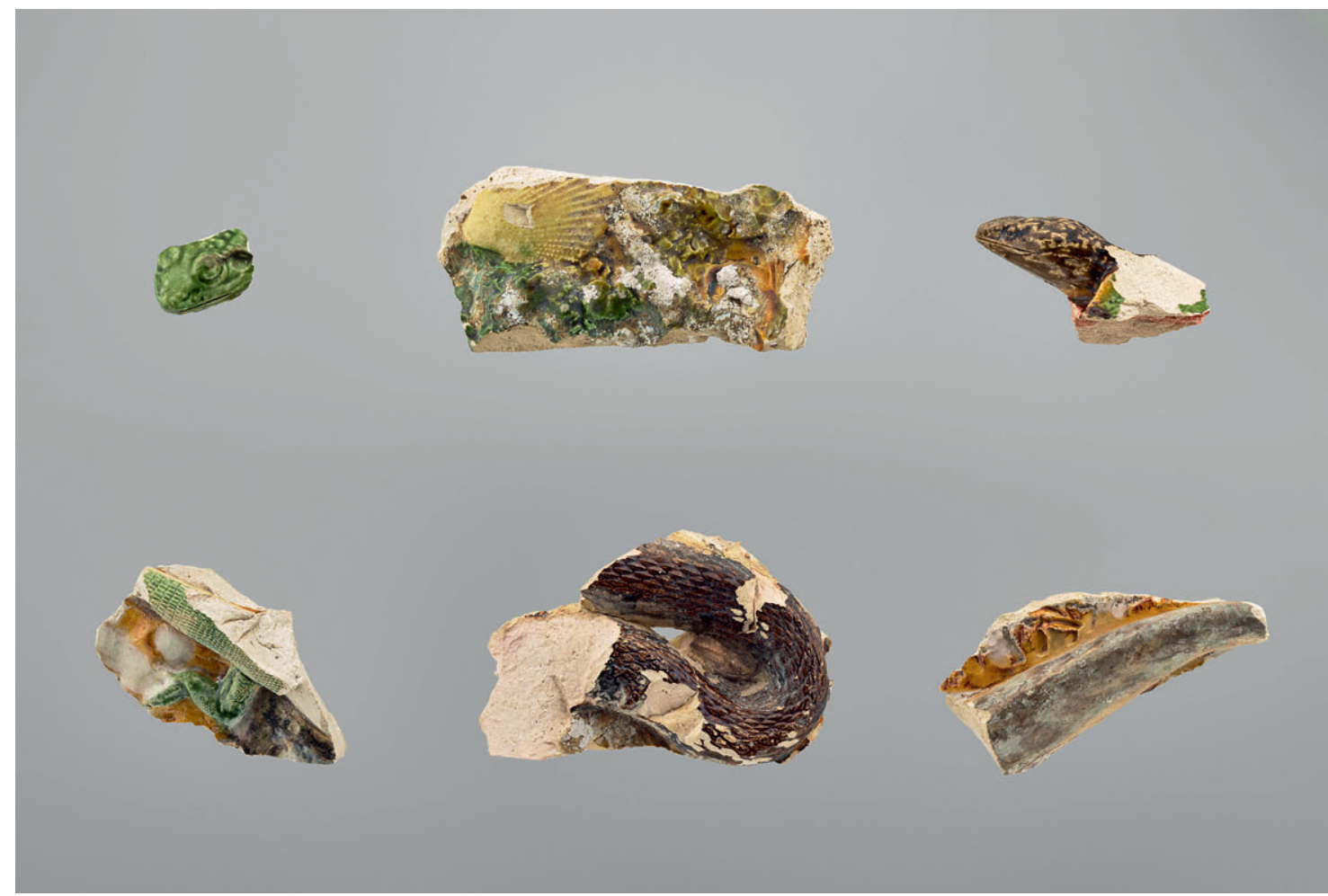

Fig. 4. Différents fragments d'animaux découverts lors des fouilles des Tuileries, terre cuite glaçurée, EP 347, 121.002.17, EP 343, AC. 243.1585, AC. 243.1860, 121.002.37.

(C) RMN-Grand Palais (musée national de la Renaissance, château d'Écouen)/Mathieu Rabeau. 


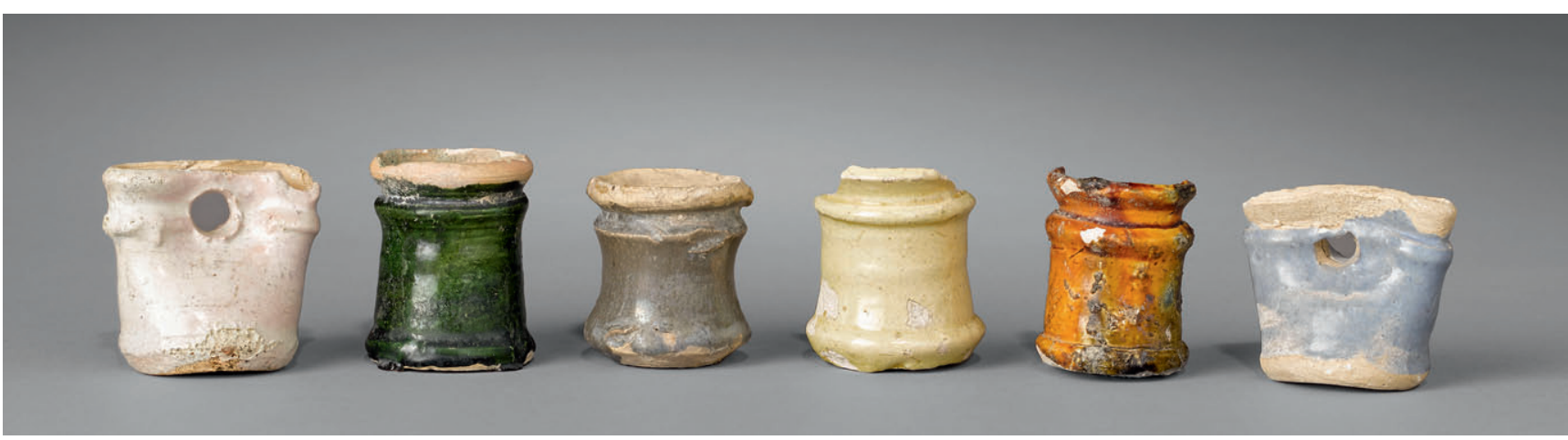

Fig. 5. Atelier de Bernard Palissy, Paris, fouilles des Tuileries, troisième quart du xvi ${ }^{\mathrm{e}}$ siècle, Essais de glaçure sur albarelles, terre cuite glaçurée, Écouen, musée national de la Renaissance. @ RMN-Grand Palais (musée national de la Renaissance, château d'Écouen)/Stéphane Maréchalle.

\section{Notes}

1. Kris, 1926.

2. Voir les articles de P. H. Smith

et A. Gerbier dans ce volume.

3. Bouquillon, 2004

4. Palissy, 2010, p. 169

5. Palissy, 2010, p. 497

6. Palissy, 2010, p. 524

7. Bouquillon et al., 2017, Castaing et Menu, 2006, Christman et al., 2004.

8. Perrin, 2001.

9. Ces grenouilles pourraient être soit attribuées à Palissy lui-même, soit correspondre à des témoins plus tardifs (début $\mathrm{XVII}^{\mathrm{e}}$ siècle) d'une fontaine réalisée par Séjourné, au moment où l'espace a été transformé en jardin. Voir Bouquillon, Doublet, 2010.

10. Mason et al., 1997, p. 56 ; Tite et al., 2008.

11. Étudiés par Solène Gilette, Caractérisation physico-chimique des glaçures plombifères transparentes de Bernard Palissy, Master I Chimie, université d'Artois, 2013.

12. Bouquillon, 2004.

13. Bouquillon et al., 2007.

14. Palissy, 2010, p. 524.

15. Majerus, Lehuédé, comm. pers.

16. Palissy, 2010, p. 495.

17. Palissy, 2010, p. 495.

18. Roisine, résultats non publiés.

19. Bouquillon et al., 2018.

20. Par exemple dans les guirlandes d'encadrement, ou dans quelques bouquets de fleurs où l'on peut trouver parfois un lézard ou un escargot, ainsi dans la lunette de Giovanni della Robbia ou sur le couvercle fleuri d'un vase conservé dans une collection privée (Cat. Exp. Boston-

Washington, 2016, Calonaci, Tripodi, 2017). 21. Archimboldo, L'eau (1556) inv.

1586, Vienne, Kunsthistorisches Museum. 22. Palissy, 2010, p. 423-424.

23. Palissy, 2010, p. 295.

\section{Bibliographie}

Bouquillon A., 2004, "Heaven and earth. 'Madonne col Bambino' and 'Rustiques figulines'", Applied physics A, vol. 79, $\mathrm{n}^{\circ} 2$, p. 161-166.
Bouquillon A., Turrell S., Robinet L., Charbonneau C., 2007, « Des glaçures Renaissance au cristal moderne. Comportement du plomb dans les verres altérés ", L'Actualité chimique, $\mathrm{n}^{\circ}$ 312-313, p. 40-46.

Bouquillon A., Castaing J., Barbe B., Paine S. R., Christman B., Crépin-Leblond T. Heuer A. H., 2017, "Lead-glazed Rustiques Figulines (Rustic Ceramics) of Bernard Palissy (1510-90) and his followers", Archaeometry, vol. 59, issue 1, p. 69-83.

Bouquillon A., Castaing J., Barbe B., CrépinLeblond T., Tilliard L., Paine S. R., Christman B., Heuer A. H. , 2018, "French Decorative Ceramics MassProduced during and after the $17^{\text {th }}$ Century: Chemical Analyses of the Glazes”, Archaeometry, doi.org/10.1111/ arcm.12349.

Calonaci S., Tripodi C., 2017, « La lunetta Antinori a Villa Le Rose : mecanatismo, ricchezza e devozione nel Rinascimento », in «Da Brooklyn al Bargello - Giovanni della Robbia, la lunetta Antinori e Stafano Arienti », Firenze, Museo Nazionale del Bargello, 10 novembre 2017-8 avril 2018, SAGEP ed., p. 14-29.

Cat. Exp. Arezzo, 2009, I Della Robbia. Il dialogo tra le Arti nel Rinascimento [Exp. Arezzo, Provincia di Arezzo, Museo Statale d'Arte Medievale e Moderna, 21 février-7 juin 2009], Gentilini G., Fornasari L. (dir.), Milan, Skira Editore, 2009.

Cat. Exp. Boston-Washington, 2016, Della Robbia. Sculpting with color in Renaissance Florence [Exp. Boston, Museum of Fine Arts, 09 août- 4 décembre 2016 et Washington, National Gallery of Art, 5 février-4 juin 2017], Cambareri M. (dir.), Boston, p. 156.

Castaing J., Menu M., 2006, "Analysis of art works and nuclear physics at the laboratory of Centre de recherche et de restauration des musées de France", Nuclear Physics News, 16, p. 4-10.
Christman B., Heuer A. H., Castaing J., 2004, "Palissy ceramics in the collection of the Cleveland Museum of Art", Technè, n 20, p. 92-95.

Heuer A., Bouquillon A., Castaing J., 2008, Bernard Palissy (1510-1590): materials Engineer "Par excellence ", Art Institute of Chicago, Northwestern University Symposium : "Productive affinities: successful collaborations between Museums and Academia”, 29-31 October 2008

Kris E., 1926, Le style rustique, le moulage d'après nature chez Wenzel Jamnitzer et Bernard Palissy. Réédition, 2005, postface de $\mathrm{P}$. Falguières, «Sur le renversement.dil maniérisme ma Macula inçaise
Paris.

Mason R. B., Tite M. S., 1997, "The Beginnings of Tin-opacification of pottery glazes", Archaeometry, vol. 39, issue 1 p. 41-58.

Palissy B., 2010, Euvres complètes, M.-M. Fragonard (dir.), seconde édition revue et annotée, Honoré Champion, Paris.

Perrin I., 2001, Les techniques céramiques de Bernard Palissy, thèse sous la direction de M. Jean Guillaume, soutenue en 1998, université Paris IV Sorbonne, Anrt, Lille, 2 vol.

Roisine G., Capobianco N., Caurant D., Wallez G., Bouquillon A., Majérus O., Cormier L., Gilette S., Gerbier A., 2017, "The art of Bernard Palissy (15101590): influence of firing conditions on the microstructure of iron-coloured high lead glazes", Applied Physics A, vol. 123, issue 8,501 , doi.org/10.1007/ s00339-017-1089-9.

Tite M., Pradell T., Shortland A., 2008, "Discovery, production and use of tinbased opacifiers in glasses, enamels and glazes from the late iron age onwards: a reassessment", Archaeometry, vol. 50, p. $67-84$.

\section{Document inédit}

Bouquillon A, Doublet C., 2010, Grenouilles de fontaine Renaissance ou de la période d'Henri IV, Rapport C2RMF no 21300, $13 \mathrm{p}$. 